\title{
Hepatitis A vaccines: How will we use them?
}

I N THE LATE '70s, PROVOST AND HILLEMAN (1) FIRST demonstrated the ability of hepatitis A virus (HAV) to replicate in cultured primate cells; this opened the way to the development of effective vaccines. Two general types of vaccines have been developed against HAV: live attenuated vaccines, which are still in the experimental phase; and inactived vaccines (Salk-type) which are about to be licensed in Canada. Two manufacturers (Merck, Sharp and Dohme, and SmithKline Beecham) have each developed an inactivated Salk-type culturederived purified vaccine; both have been shown to be highly effective in preventing hepatitis A when tested in field trials $(2,3)$. Both vaccines are highly immunogenic, even after one dose. Two doses of hepatitis A vaccine induce antibody levels which are greater than those conferred by a standard $0.02 \mathrm{mg} / \mathrm{kg}$ dosage of intramuscular gammaglobulin (4).

The duration of protection conferred by these vaccines remains uncertain. This will be determined only by continued observation in the field; but in light of the levels of antibody detected after completion of a threedose schedule it is expected that protection will last for at least five to 10 years. Furthermore, these vaccines appear able to protect against all HAV strains because all those studied to date are closely related, if not identical, antigenically. The safety profile in premarketing studies has been excellent.

The basic question for the practising clinician is: what are the indications for use of these new vaccines? HAV now is classified within the genus Hepatovirus of the family Picornaviridae. This RNA virus, an important cause of acute hepatitis, is closely related to enteroviruses and is transmitted by the fecal-oral route. However, in contrast to hepatitis $\mathrm{B}$ and $\mathrm{C}$ viruses, HAV does not establish permanent residence in infected individuals. Therefore, it is not a cause of chronic liver

All material presented in Pediatric Infectious Disease Notes has been reviewed and approved by the chairperson, Canadian Pediatric Society Board and representative members of the Canadian Pediatric Society on Infectious Diseases and Immunization disease. The number of reported cases of HAV in Canada has increased from 1533 in 1988 to 2628 in 1991. Similarly, in the United States the incidence rate of HAV, which had been declining progressively since the early '70s, rose from a low of 10 per $100,000 /$ year in 1985 to around 15 per 100,000/year in 1989. The rise in cases observed in both countries appears to be due to increased disease activity in certain high risk groups $(5,6)$. Although HAV infection does not lead to chronic liver disease, infection in the acute phase is associated with significant morbidity. Most patients with acute hepatitis A are ill for two to four weeks, missing school or work. Both prolonged cholestatic hepatitis and relapsing disease have been described. Occasionally, HAV infection may be complicated by development of fulminant hepatitis which can be fatal - the mortality rate for acute hepatitis A varies from 1.5 per 1000 children less than five years of age to 27 per 1000 adults over age 49 years.

One high risk group clearly identified as a target for hepatitis A immunization are travellers to countries where hepatitis A is endemic. Because of the excellent protection afforded by one dose of vaccine and the long term protection expected following a completed threedose schedule, these vaccines should replace immunoglobulin injections for prophylaxis of travellers. Other groups at high risk of acquiring infection which will benefit from immunization include intravenous drug users, male homosexuals, clients of institutions for the developmentally disabled and prisoners. Vaccination of some groups, such as food handlers, may also be considered because they can be efficient disseminators of infection in the community (eg, children in daycare centres which accept youngsters who are not yet toilettrained represent a target group of particular interest to the practising pediatrician). In certain American jurisdictions, daycare centres have played an important role in dissemination of hepatitis A infection into the community. The role of such centres in the spread of HAV infection in the Canadian population is unknown because it has not been well studied. The general high quality of most daycare centres in Canada may make 
this less of a risk compared with the United States. The use of hepatitis A vaccines in outbreak control currently is being studied.

Since hepatitis A does not rank high on the list of major public health problems in Canada, any decisions to recommend a universal HAV immunization program would be premature. Only when the epidemiology of the infection in Canada is better understood and when we know the duration of immunity conferred by immunization can such decisions be made nationally.

\section{REFERENCES}

1. Provost PJ, Hilleman MR. Propagation of human hepatitis A virus in cell culture in vitro. Proc Soc Exp Biol Med 1979;160:213-21.

2. Werzberger A, Mensch B, Kuter B, et al. A controlled trial of a formulin-inactivated hepatitis A vaccine in healthy children. N Engl J Med 1992;327:453-7.

3. Innis B, Snitbhan R, Kunasol P, et al. Field efficacy trial of inactivated hepatitis vaccine among children in Thailand (an extended abstract). Vaccine 1992:10

(Suppl 1):S159.

4. Fujiyama S, Iino S, Odoh $\mathrm{K}$, et al. Time course of hepatitis A virus antibody titre after active and passive immunization. Hepatology 1992;15:983-8.

5. Centres for Disease Control. Hepatitis Surveillance Report No 54. Atlanta: Centers for Disease Control, 1992.

6. Schomer K, Douglas JM, Cohn DL, et al. Hepatitis A among homosexual men - United States, Canada, Australia. MMWR 1992;41:155,161-4.

Gilles Delage, MD Montreal, Quebec 


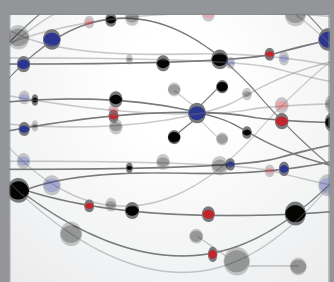

The Scientific World Journal
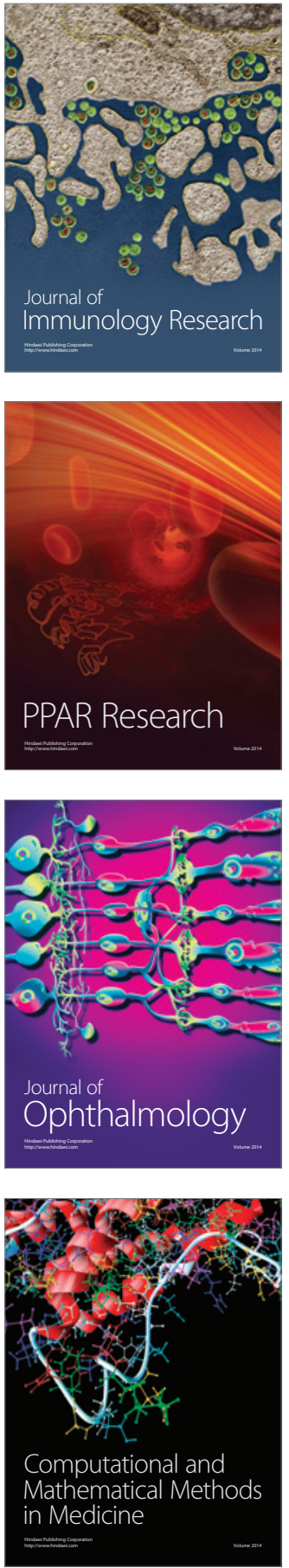

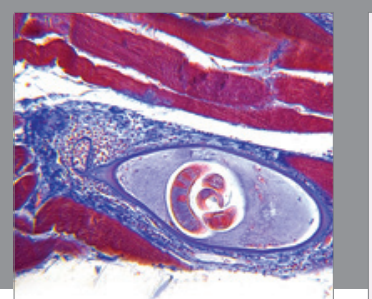

Gastroenterology Research and Practice

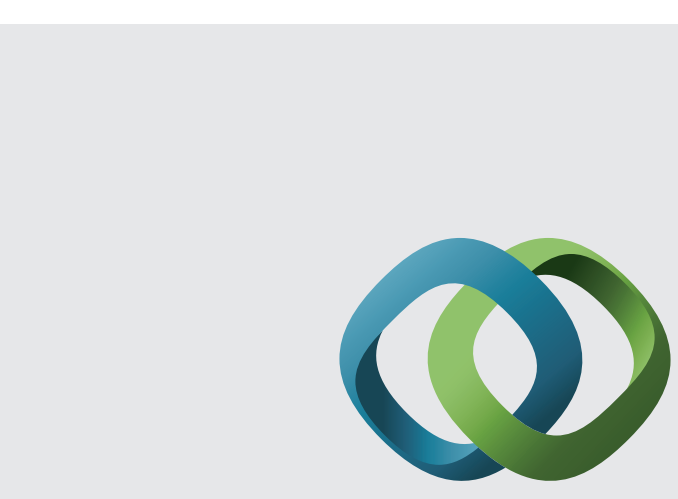

\section{Hindawi}

Submit your manuscripts at

http://www.hindawi.com
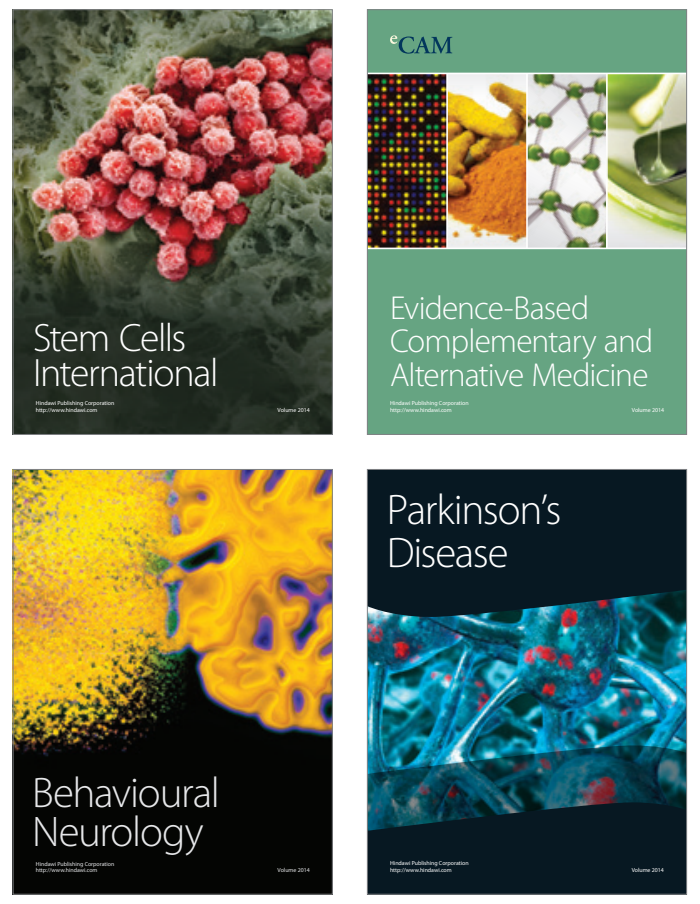
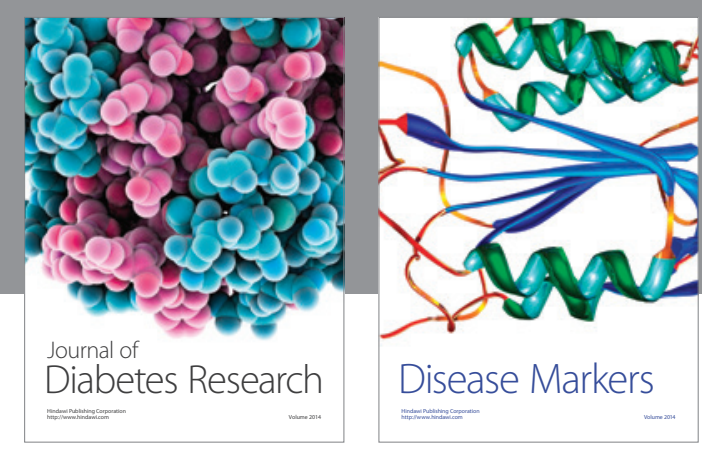

Disease Markers
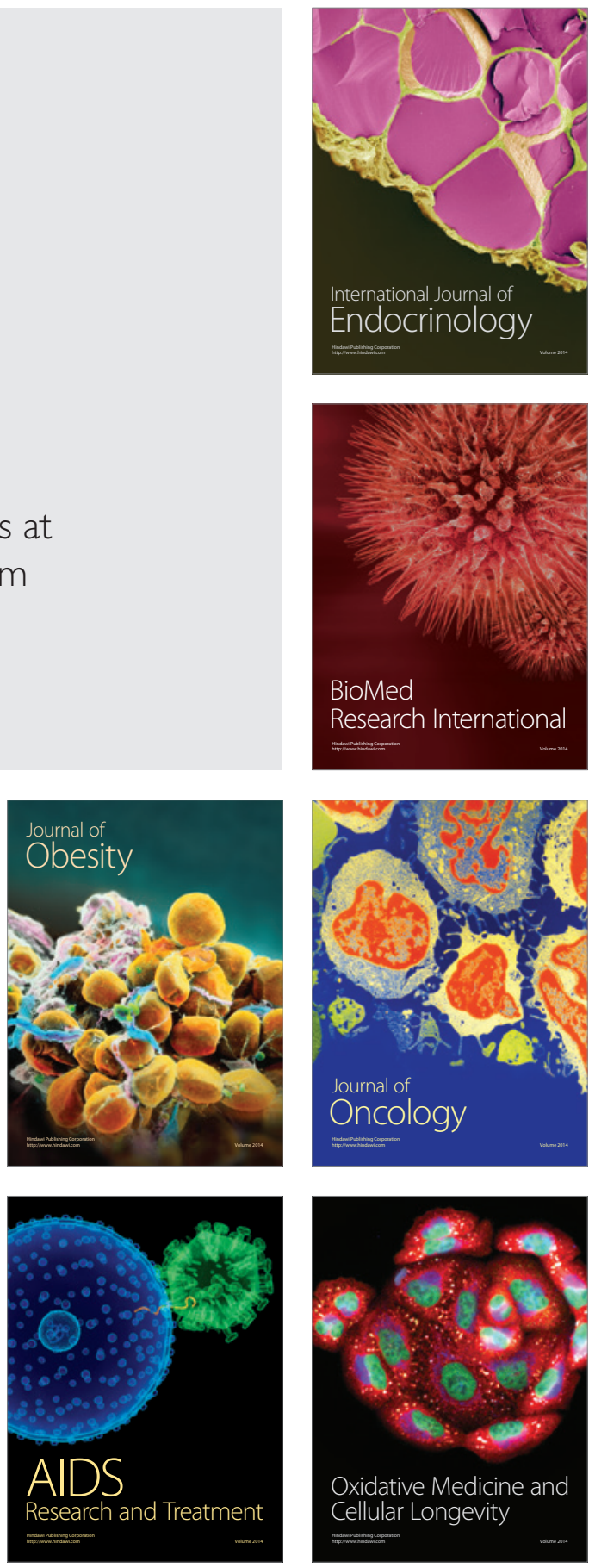